\title{
Modeling the work of a steam-water injector in a heat supply system
}

\author{
Dmitry Kitaev ${ }^{1 *}$, Egor Aralov$^{1}$, Dmitry Bugaevsky ${ }^{1}$, Artem Makarov $^{1}$ \\ ${ }^{1}$ Voronezh State Technical University, 14, Moscovskiy prospect, 394026, Voronezh, Russia
}

\begin{abstract}
One of the energy-efficient technologies is the use of injectors in heat supply systems. Steam-water injectors are very sensitive to changes in the thermodynamic parameters of water and steam. The operating range of such devices is limited to boiling liquids. The search for ranges of thermodynamic parameters that ensure the operation of the injector in real conditions of functioning of heating networks is an urgent task. The maximum energy-saving effect can be achieved by replacing devices that reduce steam pressure by injectors in which there will be a simultaneous decrease in steam pressure, heat transfer between steam and return network water to achieve the necessary thermodynamic parameters of the mixed flow. The article presents the results of modeling the operation of a steamwater injector with a cylindrical mixing chamber, taking into account the actual values of temperatures and pressures in the heating network. With constant parameters of hot steam, the possible ranges of the injector for the temperatures and pressures necessary for the implementation of temperature schedules for regulating heating networks are determined. The procedure for identifying input parameters that ensure the implementation of temperature schedules of heating networks has been carried out. Equations are obtained that allow one to determine the temperature of the water leaving the injector from the injection coefficient for the found working pressure ranges of the injected water.
\end{abstract}

\section{Introduction}

The use of steam-water injectors in heat supply systems for various purposes [1,2] is an energy-efficient technology. In jet devices, a part of the kinetic energy of one stream is transferred to another by direct contact. A feature of steam-water injectors is the location of the injected and working flow before mixing in different phase states, and then in one phase. In the process of mixing, the aggregate state of one of the flows changes. In heat supply systems with a source in the form of a steam boiler room or various types of heat and power plants in steam-water injectors, the heated medium can be network water, and the working medium is steam. During operation, the injector does not require electric energy; it is both a heat exchanger that heats water using steam energy, and a jet pump that increases the flow pressure [3]. The advantages of injectors include the absence of an external drive that consumes energy, the possibility, under certain conditions, of obtaining a resultant stream at

*Corresponding author: dim.kit@rambler.ru 
a pressure outlet more than that of the injected medium and even steam at the inlet, simplicity and reliability in operation, and environmental friendliness associated with the absence of a lubrication system, harmful radiation, and significant sound exposure. The above conditions create the prerequisites for the active introduction of such devices in various types of heat supply systems.

A significant contribution to the development of the theory of inkjet technology, engineering methods of calculation, systematization of existing knowledge and generalization of experimental data was made in the 20th century by domestic scientists N.M. Singer, A.I. Belevich, E.Ya. Sokolov and at the beginning of the 21st century Myasnikov V.E. $[4,5]$

In the studies of the authors, as a rule, steam that has undergone a preliminary pressure reduction is used in the injector as a heating medium. In modern conditions of operation of heating networks, the parameters of temperature graphs have decreased [6,7]. There are no justifications for the possibility of using injectors depending on the pressures, temperatures of the injected water and steam. Implemented technical solutions are mainly experimental, made for a specific project. This is due to the large amount of computation and the need to identify injector parameters using iterative and numerical methods for solving systems of nonlinear equations.

A standard technological solution in boiler rooms is the use of steam-water heaters for heating network water in the heat supply system. Figure 1 shows a schematic diagram of the inclusion of a steam-water heater 4 in a typical boiler room.

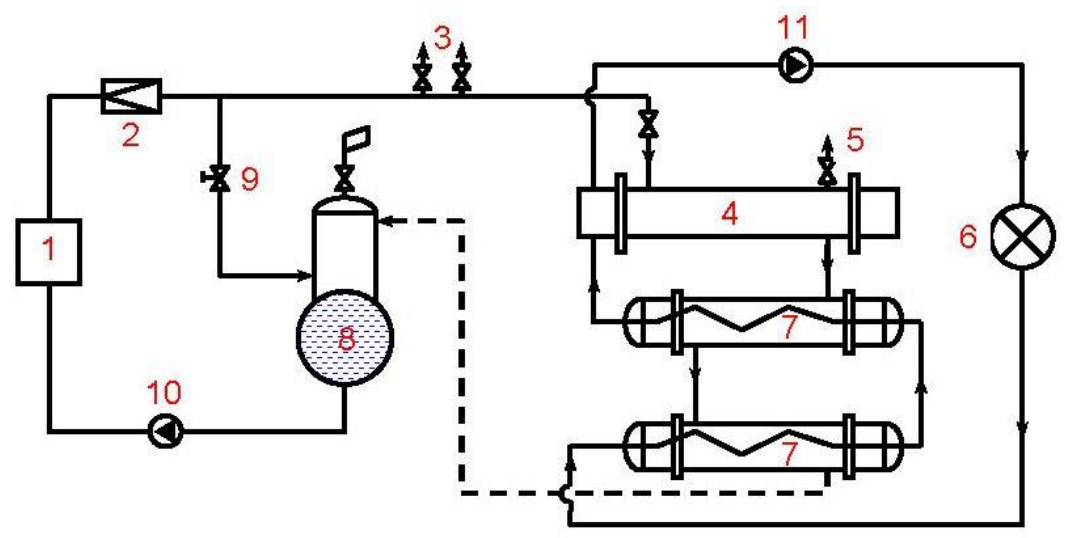

Fig. 1. Schematic diagram of the inclusion of a steam-water heater in the boiler room: 1 steam boiler; 2 - high-speed reduction installation; 3 - safety valves; 4 - steam-water heater; 5 - heater safety valve; 6 - heat consumer; 7 - water-water heat exchangers (condensate coolers); 8 - deaerator; 9 - steam pressure regulator; 10 - feed pump; 11- network pump.

The heaters 4 are designed for a working vapor pressure of 7 atmospheres, which necessitates the use of high-speed reduction and cooling units 2 . In such installations, steam is throttled to the required pressure and water is injected in order to achieve dry saturation in the steam. Behind the heaters, additional coolers of the condensing heating steam are installed, which are water-to-water heat exchangers 7, with the aim of preheating the return network water. Two heaters are usually installed in series with one heater.

The maximum energy-saving effect can be obtained by replacing the heaters with a steamwater injector, which will reduce the range of equipment and the power of reduction and cooling plants using chemically purified water. 


\section{Materials and methods}

The methodology for calculating the parameters and design dimensions of the injector with a cylindrical mixing chamber is described in detail by Russian scientists [4]. The methodology involves the use of reference data on gas-dynamic functions, water and steam properties. In order to increase the accuracy of the calculation, analytical equations were used.

The reduced isentropic steam velocity $\lambda_{p}$ was determined by the expression

$$
\lambda_{p}=\sqrt{\left(1-{\frac{p_{i}}{p_{p}}}^{\frac{k-1}{k}}\right) \frac{k+1}{k-1}},
$$

Where $k$-dry saturated steam adiabatic index [8]; $p_{i}, p_{p}$ - pressure of the injected medium and working steam, respectively, at. formula

The achievable injection coefficient $u$ under given conditions was determined by the

$$
u=\frac{C \sqrt{p_{i}-p_{s}}}{\left(p_{c}-p_{i}\right)+\left(2-\varphi_{3}^{2}\right)\left(p_{i}-p_{s}\right)}-1 .
$$

Equation (2) is solved by the method of successive approximations. Given the value $u$, the saturation pressure was found at the temperature of the heated water $p_{s}$.

From the heat balance equation, the temperature of the water in the mixing chamber $t_{m c}$ was determined by the formula

$$
t_{m c}=\frac{i_{p}+u c_{i} t_{i}}{(1+u) c_{i}}
$$

Where $c_{i}$ - heat capacity of injected water.

To determine the saturation pressure in the temperature range $60 \leq t \leq 180$ degrees Celsius, the expression obtained by the authors was used

$$
\begin{gathered}
p=1.511828 \cdot 10^{-6} t^{4}-1.9777 \cdot 10^{-6} t^{3}+2.20236 \cdot 10^{-5} t^{2} \\
-9.02455 \cdot 10^{-4} t+1.793510^{-2}
\end{gathered}
$$

The preliminary values of the minimum and maximum injection coefficients are determined from the solution of the system of equations $p_{s}=f(u), p_{m c}=f(u)$. The steam-water injector has its own operating range, determined by the minimum and maximum injection coefficients $u_{\min }$ and $u_{\text {max }}$. With a decrease in the flow rate of injected water and a constant flow rate of steam, the temperature can rise to saturation at a pressure in the mixing chamber, and the injector breaks down due to a lack of water for steam condensation. This mode defines $u_{\text {min. }}$. With an increase in the injection coefficient, the temperature in the mixing chamber drops, and at the same time, due to an increase in the water velocity, the pressure drops. At a certain value in the inlet section of the mixing chamber, the pressure may drop to the saturation pressure at the temperature of the heated water $p_{s}$. Boiling water may occur. This mode is determined by the $u_{\max }$ value. The injector ranges from $u_{\min }$ to $u_{\max }$. Determining the operating ranges of the injector and the corresponding values of the temperatures and pressures at the outlet is the central task of the work. 
The dependence of the pressure at the beginning of the mixing chamber $p_{2}$ on the mixing coefficient has the form

$$
p_{2}=p_{i}-\frac{k}{2}\left(\frac{2}{k+1}\right)^{\frac{k+1}{k-1}}\left(\frac{f p_{*}}{f_{3}}\right)^{2} \frac{v_{i}}{v_{p}} p_{p}(1+u)^{2}
$$

The final values of the injection coefficients were determined by solving the system of equations $p_{s}=f(u), p_{2}=f(u)$.

In the calculations, the vapor pressure at the outlet of the boiler was taken equal to 13 at, the temperature and pressure of the injected water, respectively, 70 degrees Celsius and 7 at. The design pressure after the injector is 10 at.

\section{Results}

The results of calculations of the values of the minimum $u_{\operatorname{mim}}$ and maximum $u_{\max }$ of the injection coefficient and the corresponding condensation pressures $p_{s}$ and at the outlet of the injector $p_{c}$, as well as the corresponding temperature, at 3,2 $\leq p_{i} \leq 7 \mathrm{at}$, are presented in the table.

Table 1. Final calculated values.

\begin{tabular}{|c|c|c|c|c|c|c|c|c|}
\hline $\mathbf{p}_{\mathbf{i}}$ & $\mathbf{p}_{\mathbf{s}}\left(\mathbf{u}_{\mathbf{m i m}}\right)$ & $\mathbf{u}_{\operatorname{mim}}$ & $\mathbf{p}_{\mathbf{s}}\left(\mathbf{u}_{\max }\right)$ & $\mathbf{u}_{\mathbf{m a x}}$ & $\mathbf{t}\left(\mathbf{u}_{\operatorname{mim}}\right)$ & $\mathbf{t}\left(\mathbf{u}_{\max }\right)$ & $\mathbf{p}_{\mathbf{c}}\left(\mathbf{u}_{\mathbf{m i m}}\right)$ & $\mathbf{p}_{\mathbf{c}}\left(\mathbf{u}_{\mathbf{m a x}}\right)$ \\
\hline 3.2 & 1.891 & 11.347 & 1.7226 & 12.1016 & 118.2 & 115.43 & 10.163 & 9.962 \\
\hline 3.7 & 2.6405 & 9.081 & 1.4628 & 13.649 & 129.04 & 110.63 & 11.375 & 9.943 \\
\hline 4 & 2.994 & 8.3942 & 1.3988 & 14.1092 & 133.35 & 109.39 & 11.873 & 9.932 \\
\hline 5 & 4.0452 & 7.037 & 1.3123 & 14.7946 & 144.05 & 107.68 & 13.227 & 9.904 \\
\hline 6 & 5.009 & 6.2573 & 1.3134 & 14.7854 & 152.01 & 107.7 & 14.373 & 9.878 \\
\hline 7 & 5.9133 & 5.7415 & 1.3668 & 14.3529 & 158.28 & 108.76 & 15.383 & 9.853 \\
\hline
\end{tabular}

It should be noted that the pressure of the injected water, taken in the calculations of 7at, in the conditions of operation of heating networks is overstated. Typically, the pressure according to the strength condition of cast-iron heating devices should be no more than 6 at [9.10]. By analogy, calculations were performed for $3 \leq p_{i} \leq 6$ at. The calculation results for $u_{\min }$ and $u_{\max }$, which determine the extreme values of the injector operating range, are given in the table. The minimum value of the pressure of the injected water was determined, which ensures the absence of cavitation during the operation of the injector, which has a value of 3,1 at.

Figure 2 shows the values $p_{s}=f(u)$ и $p_{2}=f(u)$ for the corresponding $p_{i}$ at the intersection of which the minimum and maximum injection coefficients for the working area are determined. Figure 3 shows the pressure values at the outlet of the injector as a function of $u$ in the interval of the operating range for the considered $p_{i}$. Figure 4 shows the dependence of the temperature of the water at the outlet of the injector on the injection coefficient $u$ with the ranges at different pressures of the injected water. 


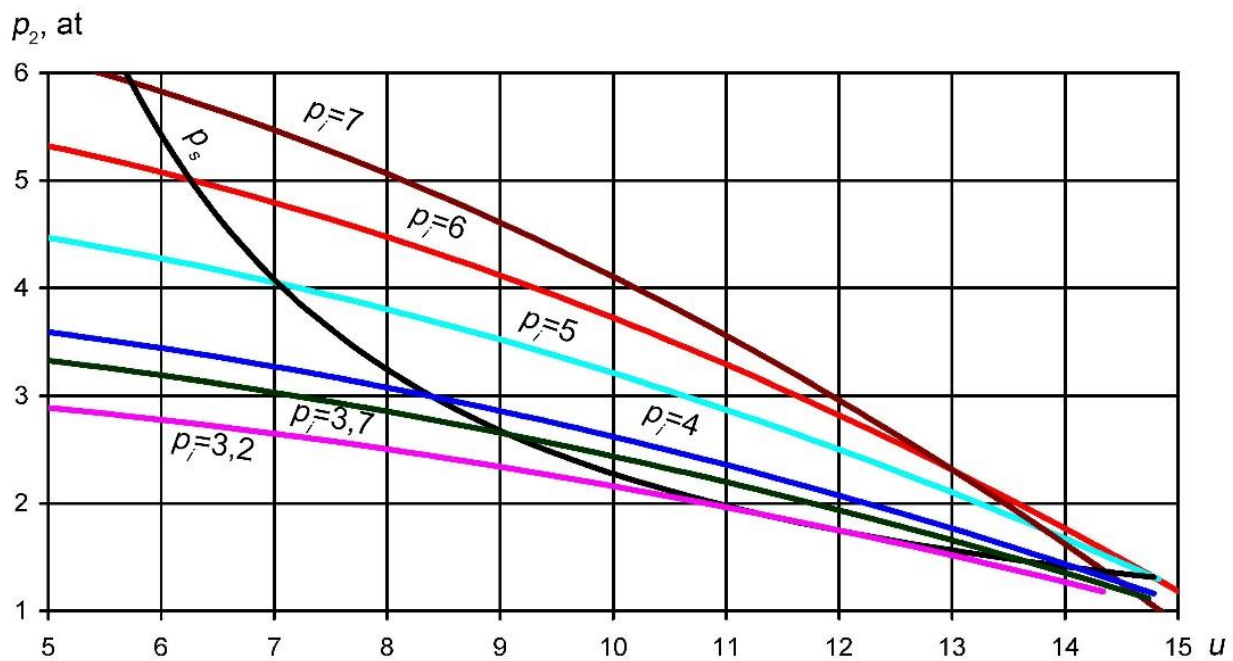

Fig. 2. $p_{s}=f(u)$ and $p_{2}=f(u)$ for $3,2 \leq p_{i} \leq 7$ at.

From figure 2 and the data of the table it follows that the working range of the injector decreases with decreasing pressure of the injected water. For $p_{i}=3,2, \Delta u=u_{\max }-u_{\operatorname{mim}}=0,7546$, and for $p_{i}=7, \Delta u=8,6114$. In practice, this means that the lower the pressure of the injected water, the smaller the range of possible regulation of the resulting flow in temperature.

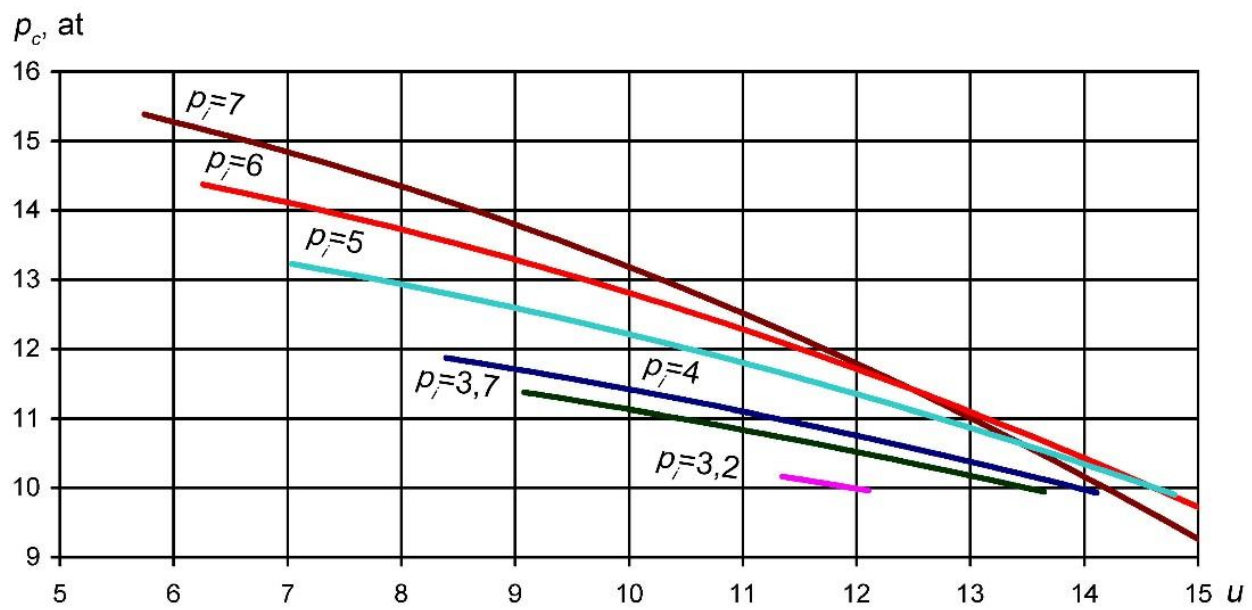

Fig. 3. $p_{c}=f(u)$ for $3,2 \leq p_{i} \leq 7$ at.

From figure 3 it follows that the pressure at the outlet of the injector varies depending on the injection coefficient. With a change in the mixing coefficient, the outlet pressure also changes, so at $p_{i}=3,2 \mathrm{at}$, the possible change in the outlet pressure will be $\Delta p=0,2$ at, at $p_{i}=3,2$ $\Delta p=1,941$ at, and at $p_{i}=7$ at $\Delta p=5,53$ at. When used in heating networks, a pressure control device is necessary [11]. During the operation of the injector, the steam condenses and as a result, the volume of water in the heat supply system will increase, which will negatively affect the hydraulic mode. When using a steam-water injector, it is necessary to remove excess water from the circuit [5].

From Figure 4 and the data of the table it follows that the temperature change increases significantly with increasing pressure: at $p_{i}=3,2 \Delta t=t\left(u_{\operatorname{mim}}\right)-t\left(u_{\max }\right)=2,77$ degrees Celsius, 
and at $p_{i}=7 \Delta t=49,52$ degrees Celsius. With increasing pressure of the injected water, the possible range of regulating the temperature of the supply water expands. Under the accepted calculation conditions, it will not be possible to implement quality control schedules [11] for heat networks in the injector over the entire range of outdoor temperatures. The minimum value of water temperature under the calculation conditions is 107,68 degrees Celsius.

$t_{c}$, deg. Celsius

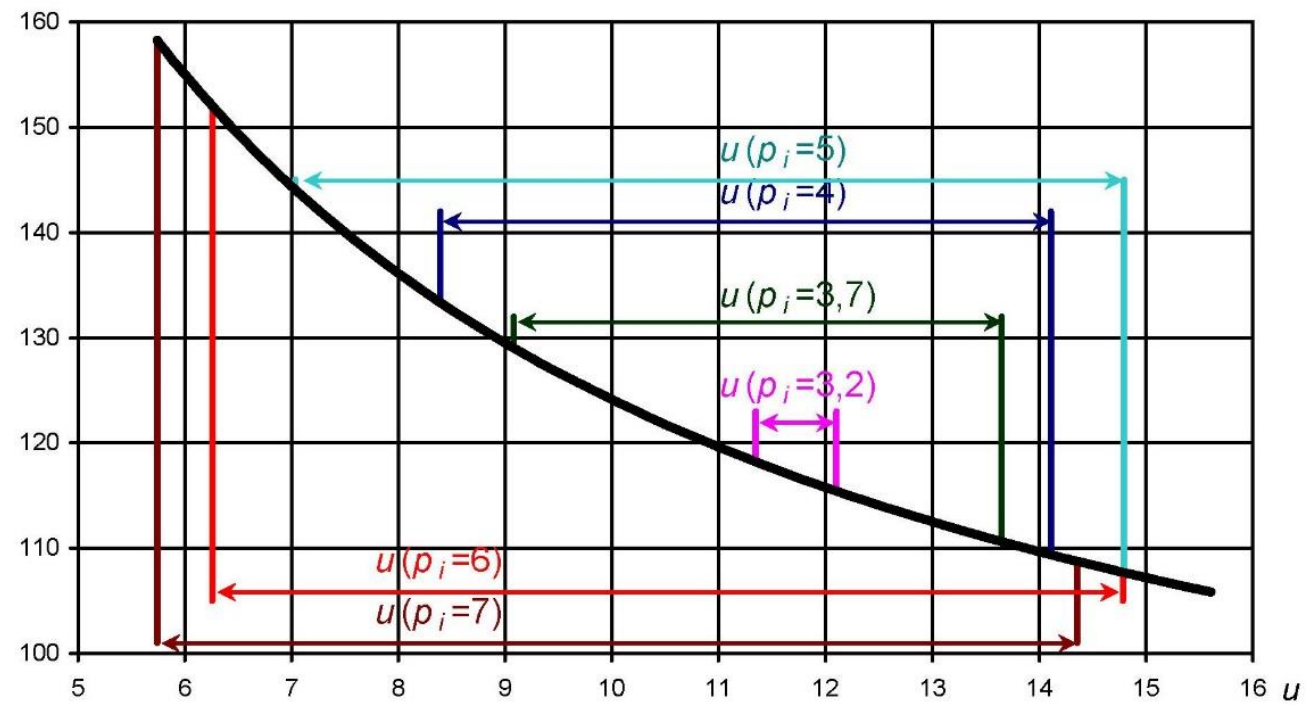

Fig. 4. $t_{c}=f(u)$ for $3,2 \leq p_{i} \leq 7$ at.

In the above calculation results, the temperature of the injected water was taken to be 70 degrees Celsius, as in the temperature graphs of quality control. At the calculated outdoor temperatures for the design of heating in the accepted calculation conditions, there are prerequisites for the implementation of schedules 110/70 and above.

\section{Discussions}

The temperature of the injected water and its pressure necessary to implement the possible temperature schedules of the heating network were determined. Typically, in heating networks with a closed circuit, the temperature of the water in the supply line is taken at least 70 degrees Celsius, i.e. it is necessary to set the values that ensure the same temperature of the water at the outlet of the injector. The temperature of the working steam at the inlet to the injector and its state is assumed unchanged.

The numerical simulation made it possible to establish that under the calculation conditions for the pressure range in the return line from 3 to 7 atmospheres, provided that the minimum outlet water temperature is 70 degrees Celsius, the temperature of the injected water can be found by the formula

$$
t_{i}=1.05 p_{i}+24.783
$$

From formula (6) it follows that in the pressure range under consideration, the temperature should be on average 30 degrees Celsius, which is a significantly underestimated value for the return line of heating networks. In practice, this means that in the range of outdoor temperatures from the beginning of the heating period to the break of the schedule, it is necessary to reduce the temperature of the water at the inlet to the injector. 
The task was set to find the necessary parameters that would allow to realize the possible temperature schedules of heating networks, taking into account the real pressure values in the return line.

The calculations showed that the output value $t_{c} \geq 70$ degrees Celsius will provide the temperature of the injected water $t_{i}=50$ degrees Celsius $=$ const, while $3 \leq p_{i} \leq 4$ at, $p_{c}=5$ at; $t_{i}=46$ degrees Celsius $=$ const, with $3,5 \leq p_{i} \leq 5 \mathrm{at}, \quad p_{c}=6 \mathrm{at}$. The dependence of the outlet water temperature tc on the injection coefficient $u$ for the two indicated pressure ranges of the injected water can be described by the equations:

$$
\begin{gathered}
t_{c}=6.488 \cdot 10^{-4} u^{4}-5.6145 \cdot 10^{-2} u^{3}+1.8268 u^{2}-27.81 u+250.874 \\
t_{c}=9.43 \cdot 10^{-4} u^{4}-7.462 \cdot 10^{-2} u^{3}+2.232 u^{2}-31.4479 u+258.127
\end{gathered}
$$

It follows from expressions (7) and (8) that when the temperature at the outlet of the injector changes in the interval $70 \leq t_{c} \leq 150$ degrees Celsius, according to equation (7), 29.49 $\geq u \geq 5.066$, and according to equation (8), $24.303 \geq u \geq 4.858$.

\section{Conclusion}

The thermodynamic parameters of the steam-water injector calculated for installation in a steam boiler to replace steam-water heaters were calculated. The injector operating range is determined under the calculation conditions, which is at injection coefficients from 5,731 to 14,385 .

Based on the calculation results, it was found that under the conditions of the initial data, the steam-water injector in the heat supply system can be used only for the range of outlet water temperatures from 109 to 150 degrees Celsius. To obtain lower temperatures, it is necessary to use multi-stage injection, or additional devices for mixing the flows of working steam and water.

The conducted studies made it possible to determine the parameters of the injected water, which make it possible to implement temperature schedules for regulating water heating networks in a steam-water injector with a cylindrical mixing chamber, at a constant pressure and temperature of dry saturated steam at the inlet. The possibility of using a single-stage steam-water injector using steam pressure of 13at without preliminary reduction of its parameters is established. However, for the implementation of such an event, it is necessary to use additional equipment that regulates the temperature and pressure of the injected and network water, as well as the withdrawal of excess from the network circuit.

\section{References}

1. K.E. Aronson, A.Yu. Ryabchikov D.V. Brezgin, I.B. Murmansk, Steam Turbine Condensing Unit Ejectors (Yekaterinburg, 2015)

2. K.E. Aronson, A.Yu. Ryabchikov, Yu.M. Brodov, D.V. Brezgin, N.V. Zhelonkin, I.B. Murmansk, Heat Power Engineering 2, 28-35 (2017)

3. N.S. Lupov, Omsk Scientific Herald 2(140), 167-168 (2015)

4. E.Ya. Sokolov, N.M. Singer, Inkjet Apparatus (Moscow, 1989)

5. V.E. Butchers, Steam and water injectors. Calculation, design, application, 100 design options (St. Petersburg, 1997)

6. N.N. Ponomarev, Proceedings of the Krylovsk State Scientific Center 2(380), 101 - 110 (2017)

7. D.N. Kitaev, Industrial Energy 7, 34-37 (2013) 
8. N.M. Tsirelman, Technical Thermodynamics (St. Petersburg, 2019)

9. D.N. Kitaev, T.V. Schukin, Energy Saving 6, 59 - 63 (2012)

10. D.N. Kitaev, A.V. Zolotarev, N.S. Sixth, Science Magazine Engineering systems and structures 2(7), 26 - 29 (2012)

11. V.N. Semenov, N.S. Popov, The practical application of energy-saving technologies (Tambov, 2014) 